\title{
Investigation of interaction between $D C D C 2$ and KIAA0319 in a large German dyslexia sample
}

\author{
Kerstin U. Ludwig · Darina Roeske · Johannes Schumacher · Gerd Schulte-Körne • \\ Inke R. König · Andreas Warnke · Ellen Plume · Andreas Ziegler · Helmut Remschmidt • \\ Bertram Müller-Myhsok $\cdot$ Markus M. Nöthen · Per Hoffmann
}

Received: 21 February 2008/Accepted: 5 September 2008/Published online: 23 September 2008

(C) The Author(s) 2008. This article is published with open access at Springerlink.com

\begin{abstract}
The dyslexia susceptibility locus DYX2 (chr. 6p21-p22) harbours two candidate genes, DCDC2 and KIAA0319. In 2006, Harold et al. reported evidence for interaction between both genes. Having previously identified a risk haplotype for dyslexia in $D C D C 2$, but not KIAA0319, in German families, we also tested for interaction between this risk haplotype and KIAA0319. We found a nominally significant association for the
\end{abstract}

K. U. Ludwig · D. Roeske · M. M. Nöthen · P. Hoffmann ( $\square)$ Department of Genomics, Life and Brain Center, University of Bonn, Sigmund-Freud Str. 25, 53105 Bonn, Germany

e-mail: p.hoffmann@uni-bonn.de

D. Roeske · B. Müller-Myhsok

Max-Planck Institute of Psychiatry, Kraepelinstr. 2-10, 80804 Munich, Germany

J. Schumacher · M. M. Nöthen · P. Hoffmann Institute of Human Genetics, University of Bonn, Wilhelmstr. 31, 53111 Bonn, Germany

G. Schulte-Körne

Department of Child and Adolescent Psychiatry and Psychotherapy, University Hospital Munich, Pettenkoferstr. 8a, 80336 Munich, Germany

\section{R. König · A. Ziegler}

Institute of Medical Biometry and Statistics, University Hospital Schleswig-Holstein-Campus, Ratzeburger Allee 160 Haus 4,

23538 Lübeck, Germany

A. Warnke - E. Plume

Department of Child and Adolescent Psychiatry

and Psychotherapy, University of Würzburg,

Füchsleinstr. 15, 97080 Würzburg, Germany

H. Remschmidt

Department of Child and Adolescent Psychiatry

and Psychotherapy, University of Marburg,

Hans-Sachs-Str. 4, 35039 Marburg, Germany quantitative dimension "word reading", the core phenotype in the study of Harold et al., which may be considered as supportive evidence.

Keywords DYX2 - Doublecortin domain - Interaction · Reading $\cdot$ Spelling

Dyslexia is amongst the most common neurodevelopmental disorders with a prevalence of 5-12\% depending on the applied diagnostic criteria (Schumacher et al. 2007). According to ICD-10, dyslexia is "a disorder manifested by difficulty learning to read despite conventional instruction, adequate intelligence and sociocultural opportunity" (WHO 1993). Longitudinal studies have shown that it involves an extremely stable developmental disturbance which does not, in contrast to popular opinion, disappear with adolescence (Shaywitz et al. 1999).

In order to identify regions that confer susceptibility for dyslexia, results from several independent linkage studies have pointed towards a susceptibility locus on chromosome 6p21-p22 (DYX2) (for review Schumacher et al. 2007). This region harbours two independent gene clusters in close proximity to one another, namely $V M P / D C D C 2 /$ KAAG1 and KIAA0319/TTRAP/THEM2. Both regions have received support from different independent samples (Cope et al. 2005; Deffenbacher et al. 2004; Francks et al. 2004; Meng et al. 2005; Schumacher et al. 2006) and it has been concluded that of the candidate genes discussed to date, the evidence for DCDC2 and KIAA0319 is the most convincing (Fisher Francks 2006; McGrath et al. 2006; Schumacher et al. 2007; Williams O'Donovan 2006). Their involvement in the development of dyslexia has been further strengthened by functional analyses which showed that inhibition of each of the two genes, DCDC2 and KIAA0319, leads to 
poorer neuronal migration in the neocortex of fetal rats through RNA interference methods (Meng et al. 2005; Paracchini et al. 2006). However, as a limiting fact it should be noted that despite favourable findings, negative associations have been reported for both genes (Cope et al. 2005; Deffenbacher et al. 2004; Francks et al. 2004; Meng et al. 2005; Schumacher et al. 2006). Importantly, only a single study to date has sufficiently covered both genes at the same time, which is necessary to understand the relative contribution of both genes and to identify possible interactions between them. Harold et al. (2006) reported a combined analysis of the KIAA0319 and DCDC2 genes in two large samples from the UK (Cardiff and Oxford sample). While the results of this study strongly supported their previously observed association with KIAA0319, no support was obtained for $D C D C 2$. Interestingly, weak evidence was obtained for an interaction between the two loci when analysing a combined case-control set from both samples.

We have previously reported strong association of the $D C D C 2$ gene with dyslexia in severely affected German individuals (Schumacher et al. 2006). In the same study we did not obtain any evidence for a contribution of the KIAA0319 gene, however, we did not include the markers that Harold et al. (2006) reported as the most strongly associated markers in their recent study. Consequently, we expanded our study to include those markers in order to obtain a comprehensive picture of the contribution of KIAA0319 to the development of dyslexia in the German population. Our sample consisted of 244 German families with a severely affected child showing a discrepancy of at least 2 SD between expected and observed spelling score. The inclusion criteria and phenotypic measures have been previously described (Schulte-Körne et al. 2007). Genotyping was performed using the iPlex-assay by
Sequenom ${ }^{\mathrm{TM}}$, and analytes were measured with a MALDITOF (matrix-assisted-laser desorption/ionisation time-offlight) mass spectrometry system (Bruker Daltonics). Data were analysed using the SpectroTyper v3.1 software provided by Sequenom. Cluster positions were manually checked by two different members of the lab. In ambiguous situations, data points were excluded. Additionally, we included four duplicate samples to ensure data quality. They did not show any inconsistencies.

None of the six markers in KIAA0319 showed significant association with dyslexia or one of the subdimensions, neither for the entire data set nor when restricting the analysis to the most severely affected patients $(\mathrm{SD} \geq 2.5$; $n=114$; Table 1). When testing for interactions applying the multiplicative (allelic) model using UNPHASED 3.0.6 between markers in KIAA0319 and our previously identified risk haplotype in $D C D C 2$ [rs793862-rs807701 (A-C)] (Schumacher et al. 2006) we focussed the analysis on KIAA0319-SNPs rs4504469 and rs761100, as these SNPs had yielded significant evidence for interaction in Harold et al. (2006). As shown in Table 1, we obtained no significant interaction for dyslexia itself, but could identify a nominally significant result for the subdimension word reading (rs761100; $P=0.0351$ ). This may be seen as evidence for interaction between KIAA0319 and DCDC2. However, an effect of KIAA0319 alone, as reported for the UK samples, is not found in the German population.

Currently, it is not easy to interpret these discrepant findings. It is rather unlikely that different genetic factors would exist in closely related populations such as those found in the UK and in Germany, especially when considering that the results suggest the involvement of common genetic variants. The dyslexia core phenotype, however, might differ between languages. On the cognitive level, for example, various processes such as phonological

Table 1 Results of transmission disequilibrium test (TDT) and interaction analysis

\begin{tabular}{|c|c|c|c|c|c|c|}
\hline \multirow[t]{3}{*}{ Marker in KIAA0319 } & \multirow{2}{*}{\multicolumn{2}{|c|}{$\begin{array}{l}\text { Single marker TDT analysis* } \\
\text { TDT results by criterion }\end{array}$}} & \multicolumn{4}{|c|}{ Interaction analysis ${ }^{\mathrm{a}, *}$} \\
\hline & & & \multicolumn{4}{|c|}{$D C D C 2$ risk haplotype [rs793862-rs807701 (A-C)] } \\
\hline & $\begin{array}{l}\mathrm{SD} \geq 2 \\
(n=244)\end{array}$ & $\begin{array}{l}\mathrm{SD} \geq 2.5 \\
(n=114)\end{array}$ & Dyslexia & Spelling & $\begin{array}{l}\text { Word } \\
\text { reading }\end{array}$ & $\begin{array}{l}\text { Phonological } \\
\text { decoding }\end{array}$ \\
\hline rs 2179515 & 0.543 & 0.9156 & - & - & - & - \\
\hline rs761100 & 0.3055 & 0.4859 & 0.3567 & 0.0912 & 0.0351 & 0.3437 \\
\hline rs7766230 & 0.4828 & 0.8997 & - & - & - & - \\
\hline rs17491230 & 0.0579 & 0.3757 & - & - & - & - \\
\hline rs 1555090 & 0.6346 & 1.0 & - & - & - & - \\
\hline rs 3212236 & 0.4794 & 1.0 & - & - & - & - \\
\hline rs $4504469^{b}$ & - & - & 0.0553 & 0.2334 & 0.2382 & 0.6269 \\
\hline
\end{tabular}

\footnotetext{
${ }^{a}$ All trios $(\mathrm{SD} \geq 2)$ included

b Previously included in Schumacher et al.

* $P$ values, bold if significant $(P \leq 0.05)$
} 
decoding, phoneme awareness or orthographic processing are involved in the dyslexia core phenotype (Ramus et al. 2003; Schulte-Körne et al. 2007). Languages of different transparencies, such as German and English, differently apply at least some of these distinct processes. For instance, it has been shown that grapheme-phoneme recording skills require more time to develop in less transparent orthographies (Seymour et al. 2003). Thus, it seems more plausible that language-specific effects together with different ascertainment strategies may be the basis of the observed inconsistencies across the studies. This hypothesis receives some support in this present study, since a nominally significant interaction between DCDC2 and KIAA0319 is observed for the dyslexia subdimension "word reading", which is the core phenotype of the UK studies.

Acknowledgments GSK, AW, AZ, HR, BMM and MMN were supported by the Deutsche Forschungsgemeinschaft. MMN received further support for this work from the Alfried Krupp von Bohlen und Halbach-Stiftung. The study was approved by the ethical committees of the Universities of Marburg and Würzburg. Informed consent was given by all participants or their parents if aged 14 or younger.

Open Access This article is distributed under the terms of the Creative Commons Attribution Noncommercial License which permits any noncommercial use, distribution, and reproduction in any medium, provided the original author(s) and source are credited.

\section{References}

Cope N, Harold D, Hill G et al (2005) Strong evidence that kiaa0319 on chromosome $6 \mathrm{p}$ is a susceptibility gene for developmental dyslexia. Am J Hum Genet 76:581-591

Deffenbacher KE, Kenyon JB, Hoover DM et al (2004) Refinement of the 6 p21.3 quantitative trait locus influencing dyslexia: linkage and association analyses. Hum Genet 115:128-138
Fisher SE, Francks C (2006) Genes, cognition and dyslexia: learning to read the genome. Trends Cogn Sci 10:250-257

Francks C, Paracchini S, Smith SD et al (2004) A 77-kilobase region of chromosome 6p22.2 is associated with dyslexia in families from the United Kingdom and from the United States. Am J Hum Genet 75:1046-1058

Harold D, Paracchini S, Scerri T et al (2006) Further evidence that the kiaa0319 gene confers susceptibility to developmental dyslexia. Mol Psychiatry 11:1061, 1085-1091

McGrath LM, Smith SD, Pennington BF (2006) Breakthroughs in the search for dyslexia candidate genes. Trends Mol Med 12:333341

Meng H, Smith SD, Hager K et al (2005) Dcdc2 is associated with reading disability and modulates neuronal development in the brain. Proc Natl Acad Sci USA 102:17053-17058

Paracchini S, Thomas A, Castro S et al (2006) The chromosome 6p22 haplotype associated with dyslexia reduces the expression of kiaa0319, a novel gene involved in neuronal migration. Hum Mol Genet 15:1659-1666

Ramus F, Rosen S, Dakin SC et al (2003) Theories of developmental dyslexia: Insights from a multiple case study of dyslexic adults. Brain 126:841-865

Schulte-Körne G, Ziegler A, Deimel W et al (2007) Interrelationship and familiality of dyslexia related quantitative measures. Ann Hum Genet 71:160-175

Schumacher J, Anthoni H, Dahdouh F et al (2006) Strong genetic evidence of $d c d c 2$ as a susceptibility gene for dyslexia. Am J Hum Genet 78:52-62

Schumacher J, Hoffmann P, Schmael C et al (2007) Genetics of dyslexia: the evolving landscape. J Med Genet 44:289-297

Seymour PH, Aro M, Erskine JM (2003) Foundation literacy acquisition in European orthographies. Br J Psychol 94:143-174

Shaywitz SE, Fletcher JM, Holahan JM et al (1999) Persistence of dyslexia: the Connecticut longitudinal study at adolescence. Pediatrics 104:1351-1359

WHO (1993) The icd-10 classification of mental and behavioural disorders: diagnostic criteria for research. World Health Organization, Geneva

Williams J, O'Donovan MC (2006) The genetics of developmental dyslexia. Eur J Hum Genet 14:681-689 